\title{
Proceedings
}

\section{Wearable FES Electrodes ${ }^{\dagger}$}

\section{David Tyler ${ }^{1, *}$ and Clémentine Mavridis ${ }^{2}$}

1 School of Fashion, Manchester Metropolitan University, Manchester M15 6BG, UK

2 Graduate School of Chemistry and Mechanical Engineering, Sigma Clermont, Clermont-Ferrand, 38120

Saint Egrève, France; clementine.mavridis@sigma-clermont.fr

* Correspondence: d.tyler@mmu.ac.uk

+ Presented at the International Conference on the Challenges, Opportunities, Innovations and Applications in Electronic Textiles (E-Textiles 2019), London, UK, 12 November 2019.

Published: 10 December 2019

\begin{abstract}
Functional electrical stimulation (FES) has been used to revitalise the muscles of people suffering from various kinds of injury. However, when human skin is incorporated into electrical circuits, it must not be treated as a passive component. Skin's electrical properties must be known when electrodes deliver electrical stimulation to the body, whether by hydrogel electrodes or by electrodes embedded in apparel. Failure to address this issue increases the risk of skin burns due to too high current through the skin/electrode interface. We have demonstrated that there is a relationship between electrode size and measured voltage. The rise of voltage with a reduction of electrode size can be explained by the diminution of the skin contact area with resulting higher skin/electrode impedances. Thus, finding an electrical skin model that represents the behaviour of human skin is important for circuit design and the product development process.
\end{abstract}

Keywords: skin/electrode interface; electrical skin model; FES; electrical stimulation

\section{Introduction}

E-textiles have been used to achieve a great variety of functional products. Developing textiles with embedded electrodes to stimulate human muscles requires a clear understanding of the interactions between skin and the electrodes (along with the type of stimulation, the size and type of electrodes, and the muscles that will receive electrical stimulation).

The experimental work reported here leads to a greater understanding of the effects of electrode size, and responses to the challenge of determining the electrical impedance of the skin/electrode interface. The aim has been to assess the viability of replacing hydrogel electrodes with screen-printed wearable electrodes. The viability of interest relates to the performance of the electrodes during stimulation treatments, but also the consistency of operation to ensure the safety of individuals experiencing the stimulation.

The experimental approach has been to use a Wheatstone Bridge electrical circuit incorporating a human arm with two attached electrodes [1]. This approach allows the determination of a balance between the skin/electrode system and an equivalent electrical circuit (the electrical skin model). This is when there is zero voltage across the Wheatstone Bridge. It is widely recognized that skin has capacitive properties as well as resistive properties, and both capacitor and resistor values are dependent of the condition of the skin. This inherent variability means that achieving a balance is non-trivial, and there were occasions in our experimental work where the balance condition was elusive. 


\section{Materials and Methods}

\subsection{The Commercial Stimulator}

The instrument used for all the tests undertaken was the TENS portable EV906A, as illustrated in Figure 1.

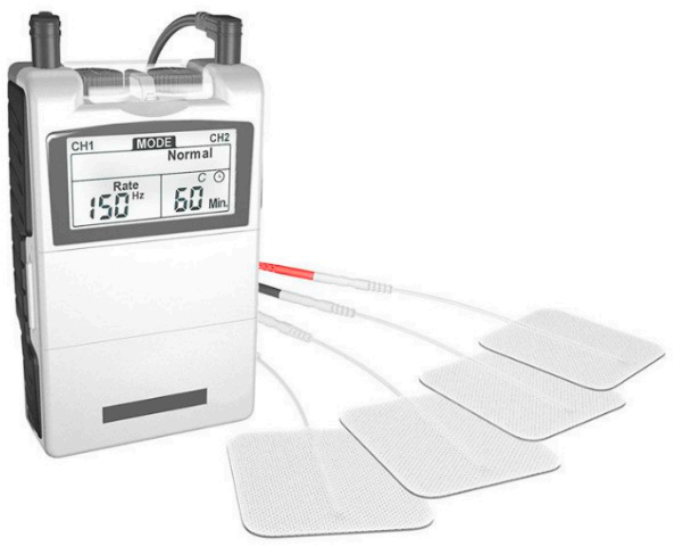

Figure 1. The TENS portable EV906A.

This unit provides asymmetrical bi-phasic square pulses, with the pulse rate adjustable from 2 to $150 \mathrm{~Hz}$, and the pulse width adjustable from 50 to $300 \mu$ s, with variable pulse amplitude.

\subsection{Measurement of Skin/Electrode Impedance Using a Wheatstone Bridge}

The circuit, based on a publication by Lawler et al. [1] and illustrated in Figure 2, balances the impedance of a human arm fitted with two electrodes and a skin/electrode model represented by resistors and capacitors. An oscilloscope was used to measure the voltages and waveforms applied to the bridge as well as the voltages and waveforms across the bridge.

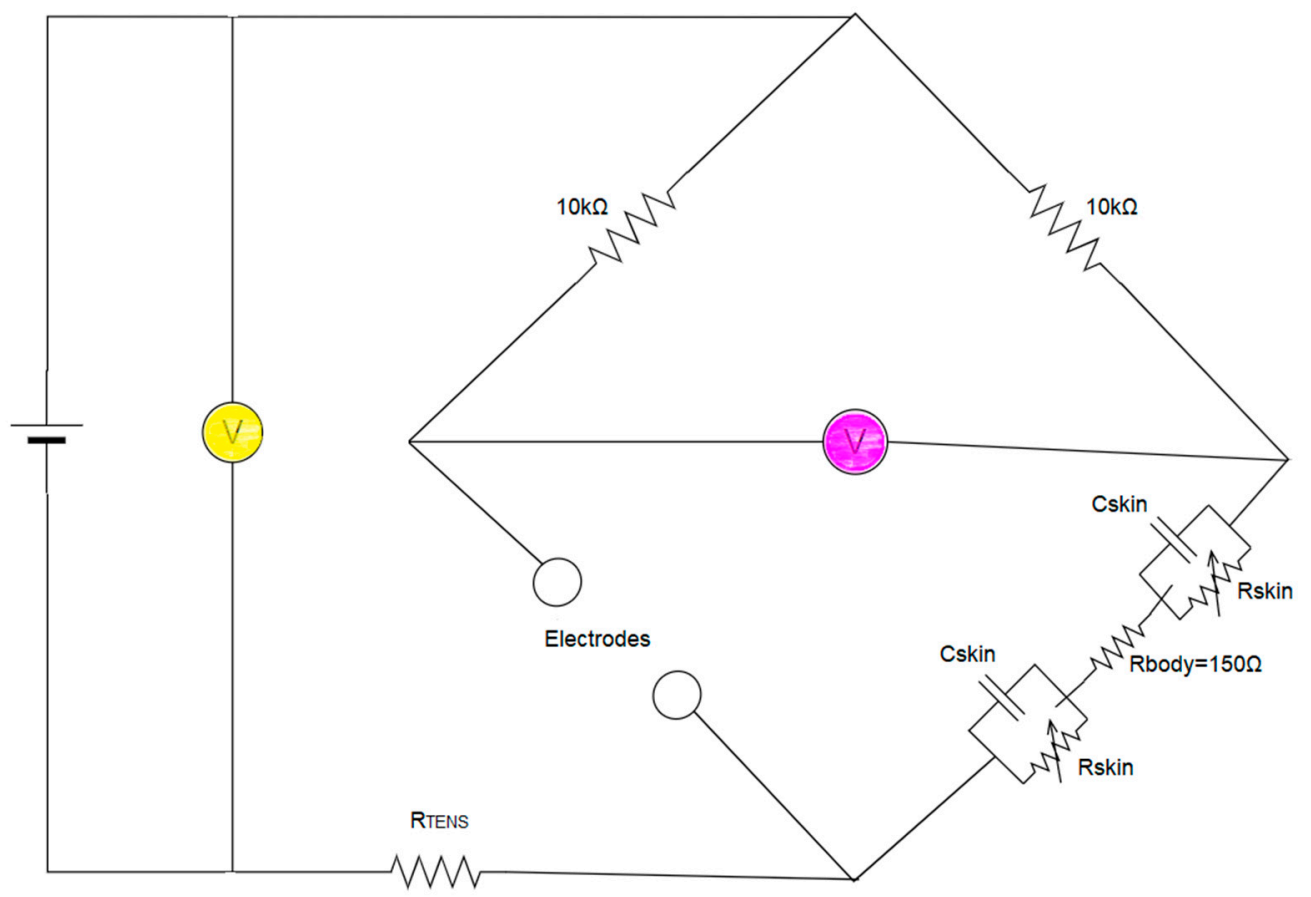

Figure 2. Wheatstone Bridge circuit. $C_{\text {skin }}$ represents the capacitive properties of skin, in parallel with

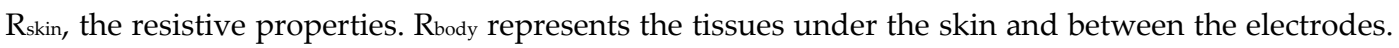
The oscilloscope recorded voltages and wave forms at $\mathrm{V}$. 


\section{Results}

\subsection{Relationship between Electrode Size and Voltage}

Reducing the electrode size is of interest when preferentially targeting specific muscles rather than stimulating many. Using a gel electrode, the area of contact of the anode was progressively reduced to obtain the results shown in Figure 3. The output of the TENS device was kept constant, and the voltages across the electrodes were recorded.

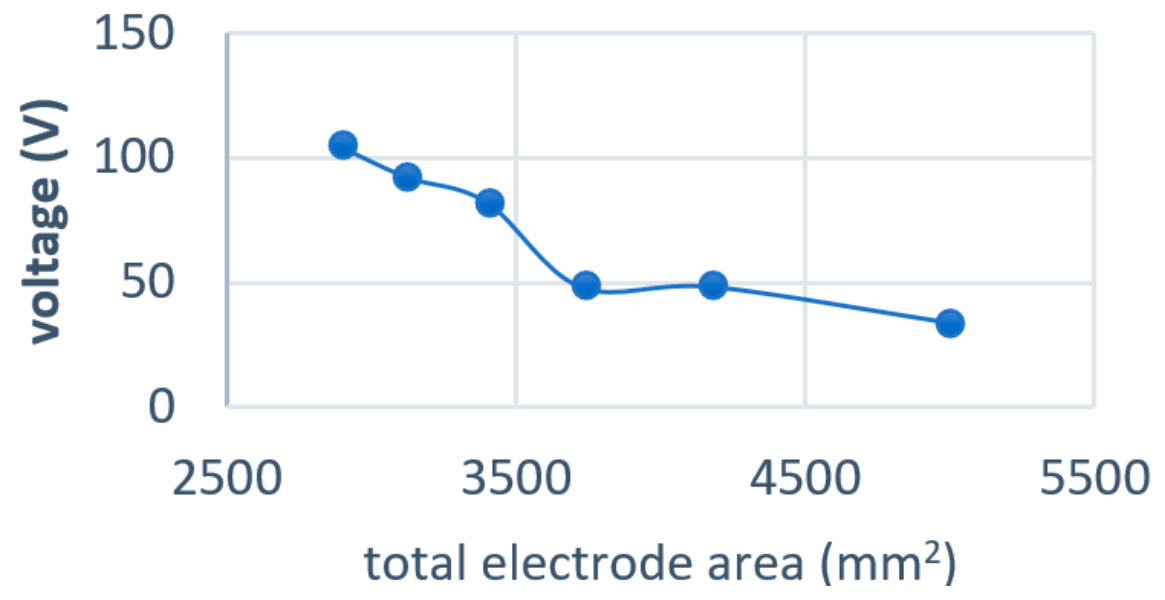

Figure 3. Voltages recorded for different gel electrode sizes (anode).

The rise of the voltage with the reduction of the electrode size can be explained by the diminution of the skin contact area with the reduction of the electrode area leading to higher electrical resistances.

\subsection{Testing Textile Electrodes}

Preliminary tests were undertaken to compare the behaviour of screen printed electrodes (as illustrated in Figure 4) to gel electrodes for different electrode sizes. These tests kept the TENS settings constant, and recorded voltages across the electrodes (Table 1). The compression exerted by the test fabric was $5 \mathrm{mmHg}$.
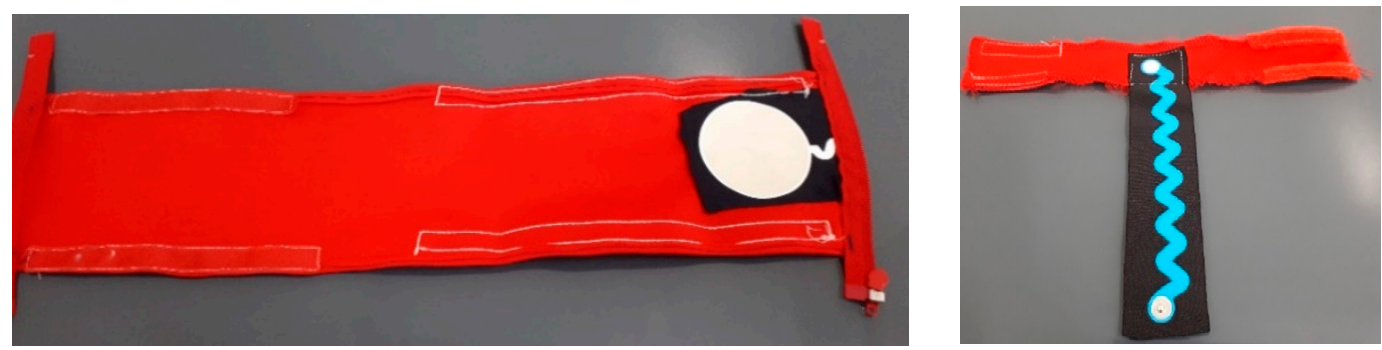

Figure 4. Large and small screen-printed electrodes.

Table 1. Preliminary test results for screen-printed electrodes.

\begin{tabular}{lccc}
\hline & Dimension Anode $\left.\mathbf{( c m}^{2}\right)$ & Dimension Cathode $\mathbf{( \mathbf { c m } ^ { 2 } )}$ & Voltage (V) \\
\hline \multirow{2}{*}{ gel electrodes (anode + cathode) } & 15 & 15 & 33.6 \\
\hline Screen printed electrode (anode) & 6 & 15 & 104 \\
and gel electrode (cathode) & 15 & 15 & 49 \\
\hline
\end{tabular}

The conclusion that can be drawn from such experiments is that the two types of electrodes have similar behaviours and performances. 


\subsection{Impedance Values for the Skin/Electrodes Assembly}

Using the Wheatstone Bridge circuit, the values of the resistors and capacitors were recorded and used to calculate the impedance of the skin/electrode assembly.

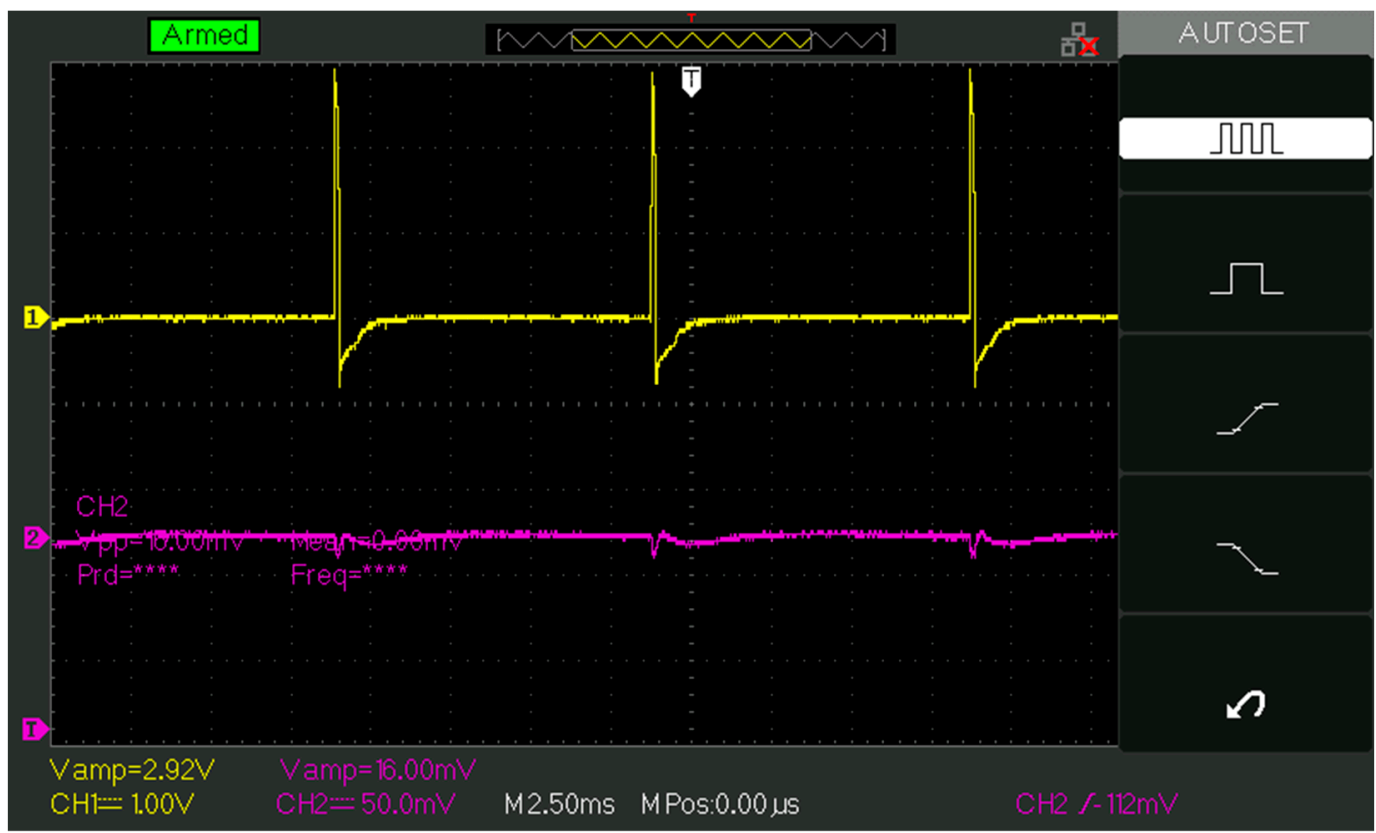

Figure 5. Oscilloscope screen-capture at balance (upper trace: TENS output; lower trace: balance condition).

At balance, illustrated in Figure 5, the impedance values obtained were approximately $11 \mathrm{k} \Omega$ for the pulsed $100 \mathrm{~Hz}$ stimulation. This figure varies with stimulation frequency because of the capacitance properties of skin.

\section{Concluding Remarks}

Further investigations are continuing with electrodes fitted to smart garments. The preliminary tests reported were undertaken to compare the behaviour of transfer printed electrodes with gel electrodes for different electrode sizes. The wearable carrying the transfer printed electrodes exerts a surface pressure of $5-15 \mathrm{mmHg}$. The initial findings are that the two types of electrode have similar behaviours and performances.

\section{Reference}

1. Lawler, J.C.; Davis, M.J.; Griffith, E.C. Electrical Characteristics of the Skin. J. Investig. Dermatol. 1960, 34, 301-308.

(C) 2019 by the authors. Licensee MDPI, Basel, Switzerland. This article is an open access article distributed under the terms and conditions of the Creative Commons Attribution (CC BY) license (http://creativecommons.org/licenses/by/4.0/). 